\title{
Automated induction thermography system for surface crack in-line inspection
}

\author{
by W. Guo*, C.Y. Ngo* and V. Kumar* \\ * Institute of Materials Research and Engineering (IMRE), A*STAR, 2 Fusionopolis Way, Singapore 138634, \\ guo_wenjiang@imre.a-star.edu.sg
}

\begin{abstract}
Compared to other active thermography techniques, induction thermography has the advantages of providing instantaneous results while being not affected by surface conditions such as emissivity or geometry. Hence, this technology has high potential to be applied for automated in-line inspection. This work will study the reflected thermal signals from a titanium sample under different moving speed. The titanium sample comes with various electrical discharge machining (EDM) notches to simulate surface cracks. Normalized temperature will be continuously calculated and plotted to reconstruct the surface condition. The final optimized configuration will be selected after leveraging the signal-to-noise ratio (SNR) and inspection speed.
\end{abstract}

\section{Introduction}

There are three most commonly applied active thermography techniques: flash thermography, induction thermography (or sometimes termed as eddy current pulsed thermography), and laser thermography [1]. As the name suggests, flash thermography makes use of flash lamps to apply instantaneous optical energy onto the surface, which converted into heat and travelled vertically into the component. The cooling pattern of the surface temperature is monitored and that reflects the subsurface condition [1]. Because of the working principle, flash thermography is mostly used for subsurface damage inspection. Besides, surface conditions such as reflectivity and contamination will affect the inspection results. Laser thermography uses a point laser source to generate a localized point heating, which laterally disperses into surrounding area. The thermal dispersion pattern will be deformed if there is a surface crack in the vicinity [1]. Hence, laser thermography is mostly used for surface damage inspection with limited resolution as the crack has to be long enough to create an impact that is significant to be captured by an infrared camera. The inspection results will also be affected by surface geometry. Unlike the prior two techniques, induction thermography uses high frequency alternating current to induce eddy current in the component, which generates heat due to Joule's Law [2]. As such, the thermal signatures are not affected by reflectivity/emissivity and is indifferent to surface geometry. In addition, sub-mm surface cracks can produce sufficient thermal contrast and thus be detected. Due to the short excitation duration and the limited number of thermal patterns need to be monitored, this technique can be easily configured for automated in-line inspection, which is a critical enabler when need to inspect large area [3]. An example of many applications will be inspecting railway tracks [3,4,5]. Last but not least, the heat is generated in the sample within the skin depth according to Eq. (1)

$$
\delta=\frac{1}{\sqrt{\pi \mu \sigma f}}
$$

where $\delta$ is the skin depth, $\mu$ is the material magnetic permeability, $\sigma$ is the electrical conductivity, and $f$ is the excitation frequency. According to Eq. (1), when using a $300 \mathrm{kHz}$ excitation frequency, the skin depth for Titanium is mostly surface and around 25mm for Carbon fibre reinforced polymer (CFRP) [5]. Therefore, induction thermography can be used for both surface and subsurface damage inspection depends on the skin depth which is only related to the material characteristics. This feature enables induction thermography to be applied for subsurface damage inspection in composite, and thus wider applications potentially.

Past research work is either focusing on the surface crack detection mechanism [6] or the speed effect [7]. This work will study systematically the detectability on a range of crack sizes under different moving speed.

\section{Methodology}

As is the case of most applications, only one-side can be accessed. Hence, induction thermography in the reflection mode will be studied in this work. The component used in this study is a titanium slab with EDM notches of different length and depth as shown below (figure 1) to simulate surface cracks. The smallest EDM notch is $0.5 \mathrm{~mm}$ in length and $0.2 \mathrm{~mm}$ in depth. The biggest EDM notch is $1 \mathrm{~mm}$ in length and $0.5 \mathrm{~mm}$ in depth. The slab will be placed on a conveyor which can run at a maximum speed of $30 \mathrm{~m} / \mathrm{min}$. Eddy current at various time interval will be activated while the thermal responses will be continuously monitored by the infrared camera. The normalized temperature will be calculated and stich together forming the thermogram to reconstruct the surface condition. The SNR under various study conditions will be one of the criteria to determine the optimized configuration. The other consideration would be the conveyor speed. After leveraging the two factors, the optimized configuration can be recommended for detecting surface cracks of various sizes. 


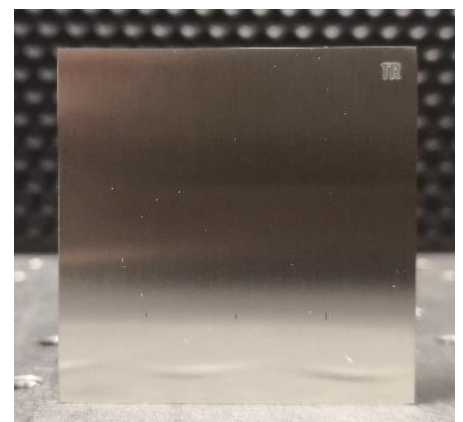

Fig. 1. Titanium slab with EDM notches to be studied

The SNR will be calculated on the reconstructed thermogram. The results will be summarized as table 1.

Table 1. SNR from various configurations

\begin{tabular}{|l|l|l|l|}
\hline Experiment index & Crack size & Conveyor speed & SNR \\
\hline 1 & & & \\
\hline 2 & & & \\
\hline$\ldots$ & & & \\
\hline
\end{tabular}

\section{Summary}

Compared to other active thermography techniques, induction thermography has advantages such as suitable for small surface crack detections, providing instantaneous results, and insensitive to surface geometry. Therefore, induction thermography has the high potential to be automated for in-line inspection of large components. This work studies the SNR of a titanium sample under different moving speed. The results will set the guideline for designing the system configuration when differently sized surface cracks need to be inspected.

\section{REFERENCES}

[1] Ciampa F., Mahmoodi P., Pinto F., et al., "Recent advances in active infrared thermography for non-destructive testing of aerospace components". Sensors, 18(609), 2018.

[2] He Y., Tian G., Pan M., et al., "An investigation into eddy current pulsed thermography for detection of corrosion blister". Corrosion Science, 78, pp. 1-6, 2014.

[3] Ehlen A., Netzelmann U., Lugin S., et al., "Automated NDT of railway wheels using induction thermography". $55^{\text {th }}$ Annual Conference of the British Institute of Non-Destructive Testing, Nottingham (UK), 2016.

[4] Wu Y., Gao B., Zhao J., et al., "Induction thermography for rail Nondestructive testing under speed effect". Proceedings of FENDT, 185-189, 2018.

[5] Netzelmann U., Walle G., Ehlen A., et al., "NDT of railway components using induction thermograpy". AIP Conference Proceedings 1706, 150001, 2016.

[6] Netzelmann U., Guo J., "Induction thermography on CFRP and the role of anisotropy", Proceedings of $14^{\text {th }}$ Quantitative InfraRed Thermography conference, Berlin (Germany), 2018.

[7] Franco L., Rodriguez F., Otero J., "Induction thermography for automatic crack detection in automotive components". Proceedings of $13^{\text {th }}$ Quantitative InfraRed Thermography conference, paper QIRT2016-165, Quebec (Canada), 2016. 